\title{
Variación de velocidad de un motor monofásico de inducción mediante el cambio de amplitud
}

\section{Speed variation of a single-phase induction motor through the change of amplitude}

CABRERA-ORNELAS, Javier†*, BRITO-MONROY, Jorge, MARTÍNEZ-MONTALVO, Anselmo y HERNÁNDEZ-NARVÁEZ, Ana Luisa

Tecnológico de Estudios Superiores de Jilotepec, México.

ID $1^{\mathrm{er}}$ Autor: Javier, Cabrera-Ornela / ORC ID: 0000-0002-9398-1498, CVU CONACY ID: IT18B386

ID $1^{\text {er }}$ Coautor: Jorge, Brito-Monroy / ORC ID: 0000-0002-3273-4872

ID $2^{\text {do }}$ Coautor: Anselmo, Martínez-Montalvo / ORC ID: 0000-0003-3404-2463

ID $3^{\text {er }}$ Coautor: Ana Luisa, Hernández-Narváez / ORC ID: 0000-0003-4500-7209, CVU CONACY ID: IT19A966

DOI: $10.35429 /$ JOIE.2020.14.4.21.26

Recibido Abril 20, 2020; Aceptado Junio 30, 2020

\section{Resumen}

Un motor de inducción es un sistema que está constituido de una parte eléctrica y una mecánica para conformar un sistema electromecánico. En estudios y análisis convencionales de los motores de inducción se centran en los valores de placa proporcionados por el fabricante el cual ya mantiene las características funcionales del motor fijas, usualmente son diseñadas mediante softwares. Los cuales proporcionan una respuesta de salida a partir de una señal de entrada. En este trabajo se propone un modelo analítico para conocer la dinámica del motor de inducción y encontrar los parámetros óptimos de algunos de sus elementos mediante el método del lugar geométrico de las raíces; Ya que ante los diversos cambios de demanda en la fabricación de productos industriales, donde los procesos de manufactura están gobernados por motores y que la velocidad del rotor se mantiene constante en intervalos de tiempo definidos, se consume la misma energía en el transcurso del proceso, por consiguiente se propone variar la velocidad para acelerar un proceso en horas pico, bajando la velocidad en ciertos tiempos, propiciando un ahorro de energía y evitando sobrecalentamiento en el motor como a continuación se describe.

\section{Abstract}

An induction motor is a system that is made up of an electrical part and a mechanical part to form an electromechanical system. Conventional studies and analyzes of induction motors focus on the plate values provided by the manufacturer which already maintains the fixed motor functional characteristics, usually designed by software. Which provide an output response from an input signal. In this work an analytical model is proposed to know the dynamics of the induction motor and find the optimal parameters of some of its elements by the method of the geometric place of the roots; Since given the various changes in demand in the manufacture of industrial products, where manufacturing processes are governed by engines and that the rotor speed is kept constant at defined time intervals, the same energy is consumed during the process, therefore it is proposed to vary the speed to accelerate a process during peak hours, lowering the speed at certain times, leading to energy savings and avoiding overheating in the motor as described below.

\footnotetext{
*Correspondencia al Autor (Correo Electrónico: javier.cabrera@ hotmail.com)

$\dagger$ Investigador contribuyendo como primer autor.
} 


\section{Introducción}

Existen diferentes aplicaciones para el control de la velocidad de un motor de inducción, en la industria surgen diariamente los retos por gestionar y optimizar costos. En los tiempos llamados set off, y los horarios de menor demanda dentro de las empresas se deben generar las menores perdidas en cuanto a costo. Este proyecto está planteado de manera práctica para conocer rápidamente la estabilidad del sistema y poder ubicar y seleccionar el nivel de voltaje que fácilmente podría cambiarse dentro de la dinámica original, también puede considerarse para la implementación de un controlador proporcional derivativo (PD) que permita cambiar automáticamente la velocidad del motor como el proceso lo requiera.

Las ventajas de emplear variadores estáticos están en el abaratamiento de costos de los componentes electrónicos de potencia. También se sabe que la forma de onda de la tensión aplicada al motor no es senoidal. Este trabajo aporta una buena base científica al estudio de los motores eléctricos. El inconveniente que se pueda suponer a esta distorsión de la forma de onda, y por tanto las perdidas adicionales a las que da lugar, se compensan sobradamente con la simplicidad del procedimiento y, sobre todo, por lo eliminación del transitorio de arranque. Hay que tener en cuenta que para potencias pequeñas (de unos pocos $\mathrm{kw}$ ), el coste del arrancador es varias veces superior al de propio motor.

\section{Metodología}

Para la implementación de un controlador dentro de la dinámica del comportamiento natural del sistema de estudio, considérese el modelo mostrado de la figura 1 .

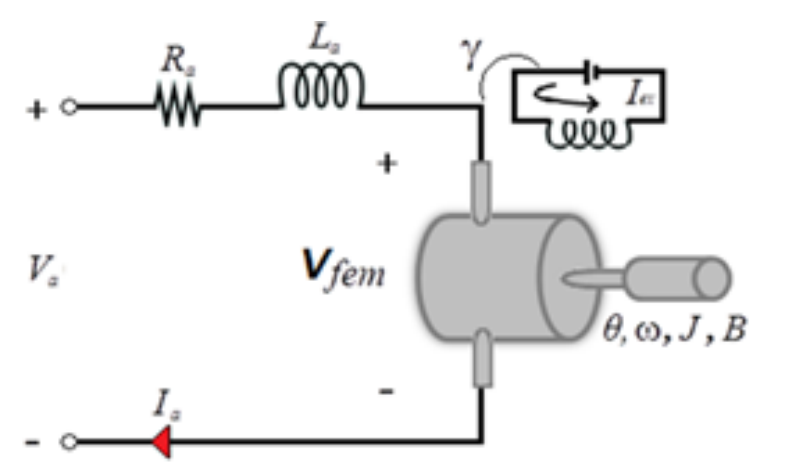

Figura 1 Diagrama esquemático de un motor de inducción Fuente: (Dorf, 2008)
Las ecuaciones que describen el comportamiento dinámico del motor de inducción mostrado en la figura 1, [Roldán, 2014], [Wildi, 2007] son:

$V_{a}=R_{a} i_{a}+L_{a} \frac{d i_{a}}{d t}+V_{f e m}$

$J \frac{d \omega(t)}{d t}=T_{r}-B \omega(t)$

Las ecuaciones (1) y (2) representan el comportamiento eléctrico y mecánico respectivamente del motor de inducción de la figura 1, donde cada uno de sus elementos se describe en la tabla 1.

\begin{tabular}{|c|c|}
\hline Símbolo & Definición \\
\hline$V_{a}(t)$ & Tensión aplicada al motor \\
\hline$I_{a}(t)$ & Corriente del motor \\
\hline$L_{a}(t)$ & Inductancia total equivalente en serie \\
\hline$R_{a}$ & Resistencia total \\
\hline$\omega(t)$ & Velocidad angular del motor. \\
\hline$J$ & Momento de inercia. \\
\hline$B$ & Coeficiente de rozamiento. \\
\hline$T_{r}$ & Par resistente. \\
\hline
\end{tabular}

Tabla 1 Variables del motor de inducción

El sistema de lazo cerrado con señal de entrada $V_{a}(S)$ y señal de salida $\omega(S)$ se ilustra en la figura 2.

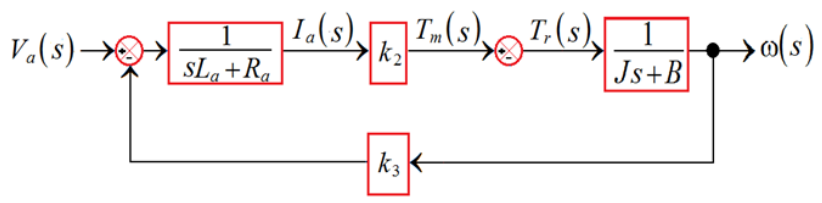

Figura 2 Diagrama de bloques de laso cerrado del motor de inducción

Para lograr el equilibrio entre la cupla eléctrica-mecánica se considera que ${ }^{T_{r}(S)=T_{m}(S)}$ obteniéndose la función de transferencia:

$\frac{\omega(s)}{V_{a}(s)}=\frac{k_{2}}{J L_{a}} \frac{1}{s^{2}+\left(\frac{J R_{a}+B L_{a}}{J L_{a}}\right) s+\left(\frac{B R_{a}+k_{2} k_{3}}{J L_{a}}\right)}$ 


\section{Método del lugar geométrico de las raíces}

La característica básica de la respuesta transitoria de un sistema de lazo cerrado se relaciona estrechamente con la localización de los polos, los polos son los elementos que permiten la solución de la función característica de la ecuación (3), entonces la estabilidad del sistema se define si la ubicación de las soluciones se grafica en el lado izquierdo del plano complejo [Dorf, 2005].

El método propuesto consiste en seleccionar adecuadamente los siguientes puntos:

- Modificar el parámetro de voltaje de entrada como una variable.

- Mantener el o los parámetros del comportamiento dinámico como constantes dentro de la función característica.

- Calcular y graficar la respuesta de salida en el tiempo de acuerdo con la variación de voltaje.

- Analizar y comparar la respuesta obtenida.

\section{Resultados}

En la tabla 2 se muestran las condiciones nominales del sistema de estudio.

\begin{tabular}{|l|l|}
\hline \multicolumn{2}{|c|}{ Valores de placa } \\
\hline$V_{a}=120 \quad$ Volt & $B=0.006$ N.m \\
\hline$I_{a}=2.5 \quad$ Amp & $K=0.57$ \\
\hline$R P M=1800$ & $R_{a}=5 \quad \Omega$ \\
\hline$T_{r}=1.14$ & $L_{a}=0.043 \quad \mathrm{H}$ \\
\hline$H P=1 / 4$ & $J=0.57 \quad \mathrm{Kg} . \mathrm{m}^{2}$ \\
\hline
\end{tabular}

Tabla 2 Condiciones nominales del sistema de estudio

De acuerdo al análisis de la ubicación de las raíces del sistema de la función característica de la ecuación (3) manteniendo como variable $\mathrm{K}$ en un intervalo, obtiene la ubicación de los polos ubicados como se muestra en la figura 3 y 4 .
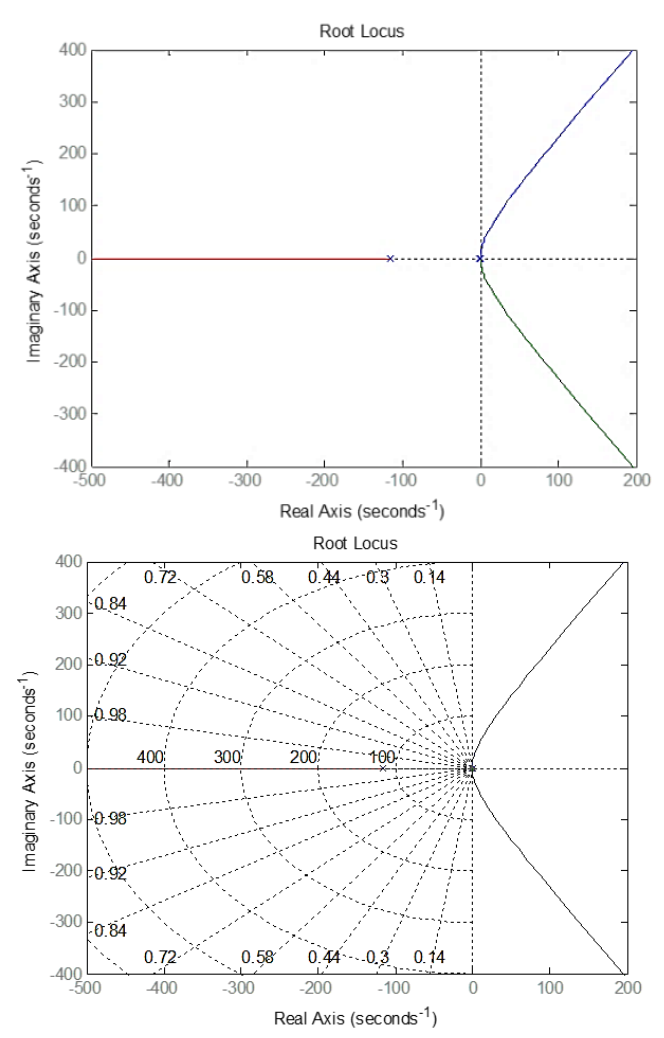

Figura 3 Tendencia de las raíces del sistema
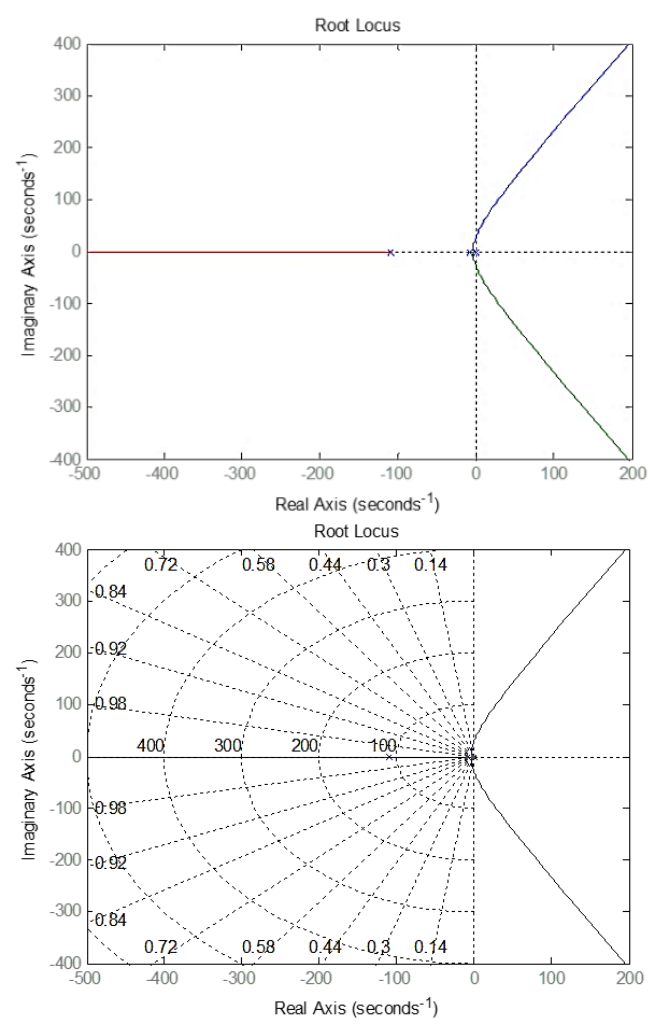

Figura 4 Identificación de valores óptimos de ${ }^{k}$ 


\section{Obtención de respuesta de salida en el dominio del tiempo}

Usando el intervalo identificado para $k \in[0.2040,0.57]$ mediante el lugar geométrico de las raíces como se muestra en la figura 4 y mediante la elaboración de diversos programas en software de Matlab se procesó las señales de respuesta en el tiempo como se ilustra en la figura 5.

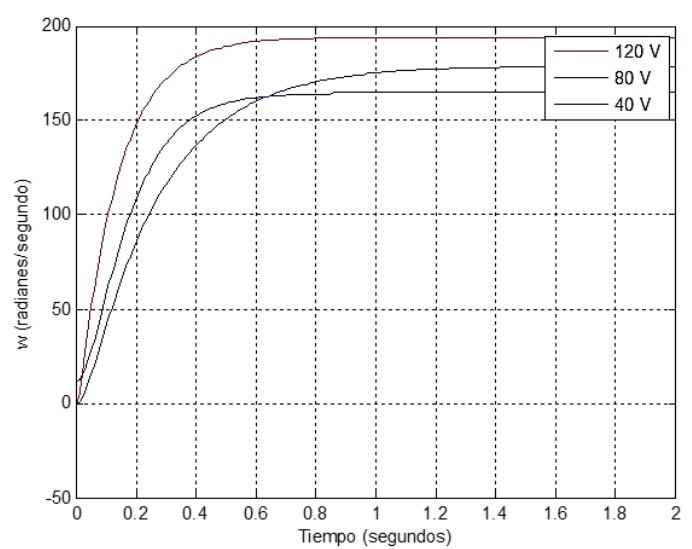

Figura 5 Respuesta en el tiempo de la $\omega$ en $\mathrm{k} \in[0.2040,0.57]$

\section{Resultados de laboratorio}

Para fines de este estudio se simula el motor de inducción de corriente alterna (c.a) a través de simulink para conocer el comportamiento del motor ante condiciones de operación de placa, la figura 6 muestra el sistema en simulink de la figura 1 .

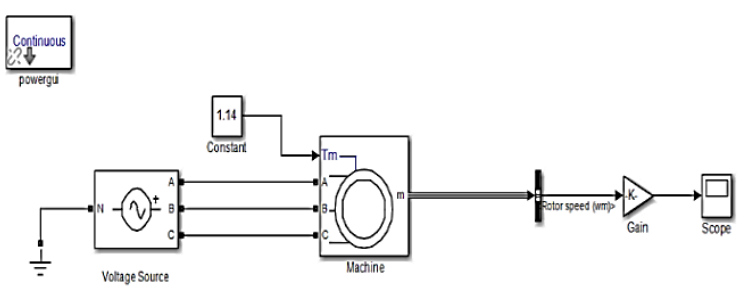

Figura 6 Diagrama del motor de inducción de ca en simulink de MATLAB

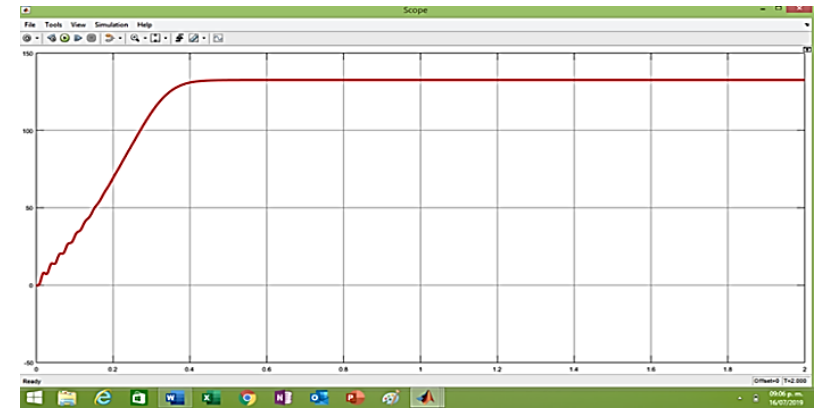

Figura 7 Velocidad del motor en condiciones nominales
Los Gráficos 1 y 2 muestran respectivamente el comportamiento grafico de la variación de voltaje/corriente y voltaje/rpm.

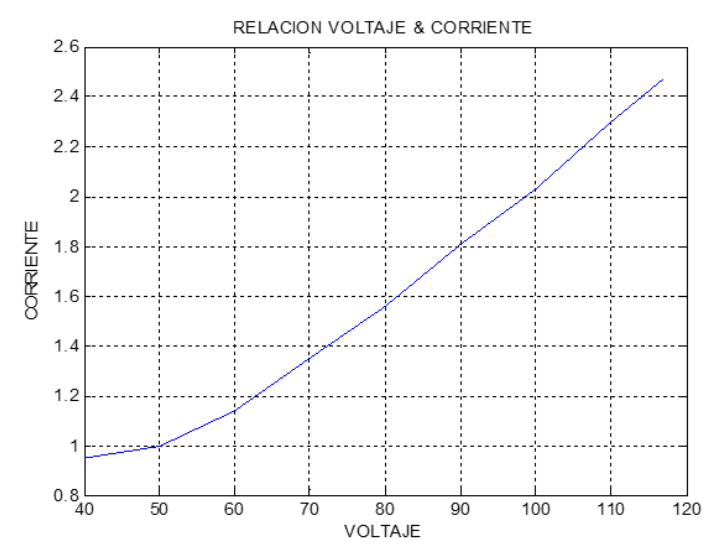

Gráfico 1 Relación voltaje/corriente

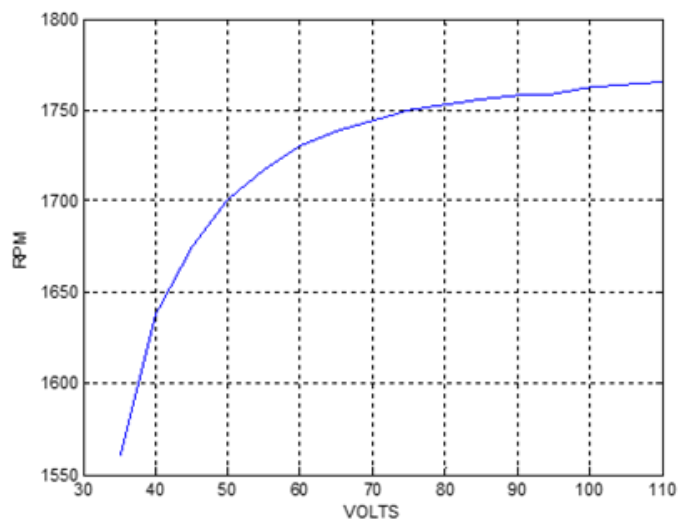

Gráfico 2 Relación voltaje/RPM

Las siguientes graficas corresponden a la variación del voltaje sobre la bobina del motor de inducción con una variación a intervalos de 5 volts en aumento las cuales fueron tomadas con osciloscopio digital en el laboratori
CABRERA-ORNELAS, Javier, BRITO-MONROY, Jorge, MARTÍNEZ-MONTALVO, Anselmo y HERNÁNDEZNARVÁEZ, Ana Luisa. Variación de velocidad de un motor monofásico de inducción mediante el cambio de amplitud. Revista de Ingeniería Innovativa. 2020 

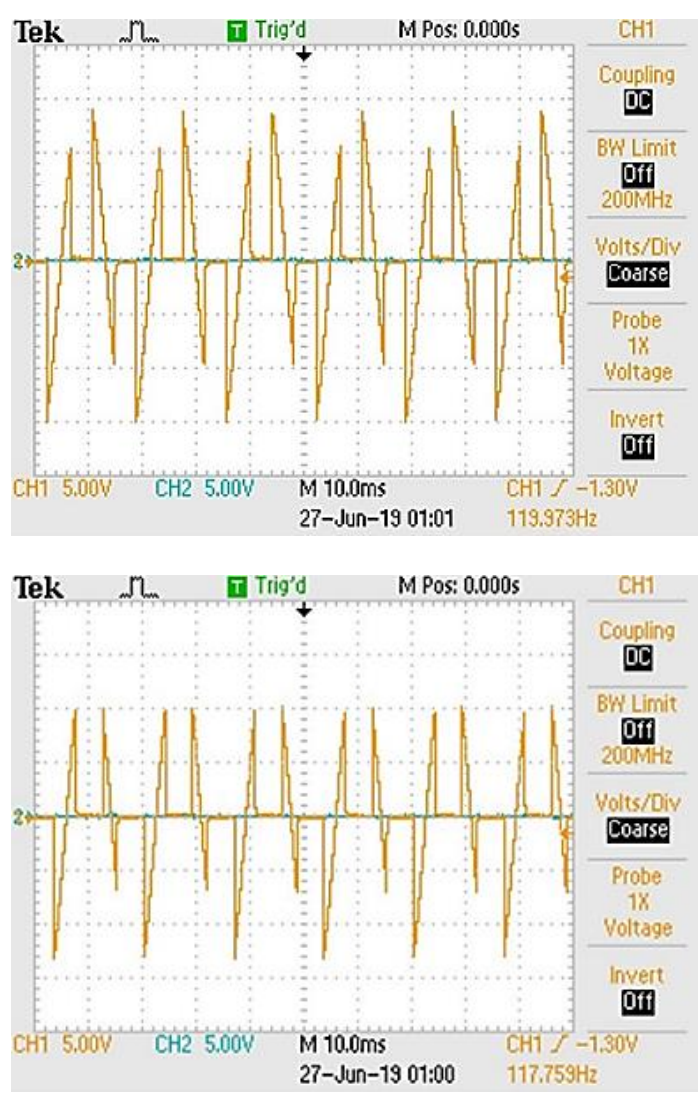

\section{Agradecimiento}

El primer autor agradece al Tecnológico de Estudios Superiores de Jilotepec por el apoyo institucional y por el uso de sus laboratorios para las pruebas necesarias para la realización de este trabajo de investigación.

\section{Conclusiones}

Existen diferentes aplicaciones para el control de la velocidad de un motor de inducción, en la industria surgen diariamente los retos por gestionar y optimizar costos. En los tiempos llamados set off, y los horarios de menor demanda dentro de las empresas se deben generar las menores perdidas en cuanto a costo. Este proyecto está planteado de manera práctica para conocer rápidamente la estabilidad del sistema y poder ubicar y seleccionar el nivel de voltaje que fácilmente podría cambiarse dentro de la dinámica original, también puede considerarse para la implementación de un controlador proporcional derivativo (PD) que permita cambiar automáticamente la velocidad del motor como el proceso lo requiera. Las ventajas de emplear variadores estáticos están en el abaratamiento de costos de los componentes electrónicos de potencia. También se sabe que la forma de onda de la tensión aplicada al motor no es senoidal.
El inconveniente que se pueda suponer a esta distorsión de la forma de onda, y por tanto las perdidas adicionales a las que da lugar, se compensan sobradamente con la simplicidad del procedimiento y, sobre todo, por lo eliminación del transitorio de arranque. Hay que tener en cuenta que para potencias pequeñas (de unos pocos $\mathrm{kw}$ ), el coste del arrancador es varias veces superior al de propio motor.

\section{Aportación}

El trabajo desarrollado propone un método alternativo para el control de suministro de energía a un circuito eléctrico de corriente alterna, la idea principal es cortar la señal senoidal del ángulo de alimentación a intervalos regulados para reducir el abastecimiento a la carga eléctrica. Esta metodología es muy fácil de adaptar a un circuito eléctrico como es el motor o resistencias eléctricas, debido a que no necesita programación como son los variadores de frecuencia comerciales.

\section{Recomendaciones}

El controlador de tensión de alterna es adecuado para algunas aplicaciones de control de velocidad. Los ventiladores y las bombas son cargas adecuadas para este tipo de control de velocidad. La eficiencia energética de este tipo de control es mala, especialmente a bajas velocidades. El gran deslizamiento que se produce a bajas velocidades provoca grandes pérdidas en el rotor. Las aplicaciones típicas son aquéllas en las que la carga es pequeña, como en los motores monofásicos con potencia igual a una fracción de caballo de vapor, o donde el periodo de operación a baja velocidad es corto.

\section{Trabajos futuros}

Se pretende escalonar este trabajo a motores trifásicos, así como el diseño del circuito electrónico.

\section{Referencias}

Richard C. Dorf, Robert H. Bishop, Sistemas de Control Moderno, 10 ed., Pearson Prentice All, S.A., Madrid, 2005. 
Róldan Viloria, Jose, Motores eéctricos, Accionamiento de máquinas, 30 tipos de motores, Paraninfa- 2005.

Theodore wild, Máquinas eléctricas y sistemas de ñpotencia, 6 ed., Pearson Printice Hall, México- 2007.

Dorf Svoboda, Circuitos eléctricos, 6 ed., Alfaomega, México-2005

Javier Sanz Feito, Máquinas Eléctricas., Pearson Prentice All, S.A., Madri, 2002.

Pedro Ponce Cruz, Javier Samplé López, Máquinas Eléctricas y técnicas modernas de control., Alfaomega Grupo Editor, S.A de C.V., México, 2010.

Bhag S. Guru, Huseyin R. Hiziroglu, Máquinas Eléctricas y Transformadores. 3ra ed., Alfaomega Grupo Editor, S.A de C.V., México, 2006.

Irving L. Kosow, Control de Máquinas electricas, Prentice Hall , España 2006.

A.E. Fitzgerald, Charles Kingsley Jr, Stephen D. Umans, Máquinas Eléctricas., 6ta ed., McGrawHill México 2005.

Katsuhiko Ogata, Sistemas de Control en Tiempo Discreto., 2da ed., Prentice Hall, México

Locón Amézquita, R. H. (2020). Detección y corrección de armónicos en inversores de energía solar, implementando sistemas embebidos en tiempo real (Doctoral dissertation, Universidad de San Carlos de Guatemala).

- González Callejas, C. (2020). Estudio de la eficacia de un tratamiento no invasivo con señales electromagnéticas por transmisión capacitiva y monopolar sobre las complicaciones postoperatorias precoces y la recuperación del estado nutricional en pacientes quirúrgicos.

- Delgado Moreno, E., Izoteco Ocampo, P. A., \& Romero Silva, J. A. (2020). Medición y corrección electrónica del factor de potencia en un sistema eléctrico monofásico.
- Payé Colquehuanca, L. (2020). Compensación de potencia reactiva y análisis de armónicos en sistemas de distribución eléctrica UNA-Puno 2018-2019.

- Rivera Vento, J. L. V., \& Romero Ruiz, W. D. (2020). EVALUACIÓN DE LA CALIDAD DE LA ENERGÍA ELÉCTRICA EN LA EMPRESA PLANET MOTOR'S SAC. 